\title{
Jochen Briesen*
}

\section{Der Urteilsbegriff und Wissen aus zweiter Hand in der Ästhetik}

https://doi.org/10.1515/dzph-2021-0052

\begin{abstract}
Although the concept of judgment has been replaced by the concept of belief in many philosophical subdisciplines, it has retained its central role in aesthetics. This paper discusses the following explanation for this: In contrast to the concept of belief, the concept of judgment presupposes conscious and first-personal engagement with the object about which the judgment is being made, and this conscious and first-personal engagement with the object in question plays a more important role in aesthetics than in other domains.
\end{abstract}

Keywords: jugdment, belief, aesthetics, second-hand knowledge

\section{Einleitung}

Im Unterschied zum Terminus „Überzeugung“ verbinden wir mit dem Terminus „Urteil“ scheinbar folgende Bedingung: Ein Urteil setzt die bewusste und erstpersonale Auseinandersetzung mit dem Gegenstand voraus, über den ein Urteil gefällt wird (vgl. dazu die Einleitung zu diesem Schwerpunkt). Ob diese Bedingung zum semantischen Gehalt des Urteilsbegriffs gehört oder beim Gebrauch des Terminus pragmatisch mitvermittelt wird, ist unklar. Im Folgenden werde ich daher lediglich davon sprechen, dass der Terminus „Urteil“ die angesprochene bewusste und erstpersonale Auseinandersetzung mit dem jeweiligen Gegenstandsbereich betont bzw. konnotiert.

In diesem Zusammenhang ist bemerkenswert: Obwohl in vielen Bereichen der philosophischen Forschung der Urteilsbegriff durch den Überzeugungsbegriff ersetzt wurde, hat er in der philosophischen Ästhetik seine zentrale Rolle beibehalten. In der entsprechenden Fachliteratur wird eher von „ästhetischen Urteilen“ (aesthetic judgments) als von „ästhetischen Überzeugungen“ (aesthetic beliefs) gesprochen. Liegt dies daran, dass im Bereich des Ästhetischen die

*Kontakt: Jochen Briesen, Freie Universität Berlin, Institut für Philosophie, Habelschwerdter Allee 30, 14195 Berlin; jochen.briesen@fu-berlin.de;

Universität Konstanz, Fachbereich Philosophie, Postfach 9, 78457 Konstanz; jochen.briesen@uni-konstanz.de 
erstpersonale Auseinandersetzung mit den Gegenständen, über die ästhetisch geurteilt wird, in stärkerer Weise vorausgesetzt wird als in anderen Bereichen?

Ein Verweis auf das ästhetische Bekanntschaftsprinzip (acquaintance principle), das häufig auf Immanuel Kant zurückgeführt wird und durch Richard Wollheim bekannt geworden ist, lässt genau dies vermuten. ${ }^{1}$

\section{Ästhetisches Bekanntschaftsprinzip:}

Eine Überzeugung der Form „X ist schön/grazil/elegant/etc.“ einer Person ist nur dann gerechtfertigt, wenn die Person selbst den Gegenstand $X$ erfahren hat.

Das Prinzip ist zumindest auf den ersten Blick nicht unplausibel. Zwar kann ich in der Annahme gerechtfertigt sein, dass ein Bild quadratisch ist, in Blautönen gemalt ist oder in einem bestimmten Museum hängt etc., ohne dass ich das Bild je gesehen hätte - weder im Original noch in einer Reproduktion. Dies ist z. B. dann der Fall, wenn ich die entsprechende Information aus einer verlässlichen Zeitung erworben habe. Aber kann ich, ohne das Bild gesehen zu haben, auch in der Annahme gerechtfertigt sein, dass es schön ist? Diese Frage wird häufig verneint. $^{2}$

Wenn das angeführte Bekanntschaftsprinzip korrekt ist und der Urteilsbegriff (im Gegensatz zum Überzeugungsbegriff) die erstpersonale Auseinandersetzung mit dem jeweiligen Gegenstand betont, dann kann verständlich gemacht werden, warum der Urteilsbegriff in der Ästhetik nicht durch den Begriff der Überzeugung verdrängt wurde. In vorliegendem Aufsatz möchte ich daher das Bekanntschaftsprinzip und sein Verhältnis zum Urteilsbegriff näher untersuchen. Ich werde dafür argumentieren, dass das Prinzip in der angegebenen Form nicht korrekt ist, aber in einer abgeschwächten Variante verteidigt werden kann. Die Verteidigung wird auf einer Analyse ästhetischer Eigenschaften beruhen, die diese als reaktions-dispositional bestimmt, d. h. als Dispositionen, in Subjekten unter bestimmten Umständen bestimmte mentale Reaktionen auszulösen. Unter Rekurs auf das schwache Bekanntschaftsprinzip werden sich dann auch Erklärungen im Hinblick auf den Urteilsbegriff in der Ästhetik anführen lassen.

Der Rest des Aufsatzes ist folgendermaßen gegliedert: Zunächst werde ich in Abschnitt 2 zeigen, dass zumindest das starke Bekanntschaftsprinzip unplau-

1 Vgl. Kant (2009), § 8; Wollheim (1980), 22.

2 In diesem Zusammenhang wird gelegentlich auch zwischen einem sprachlichen und einem epistemischen Bekanntschaftsprinzip unterschieden, vgl. z. B. Robson (2012). Für eine Diskussion des Verhältnisses beider Prinzipien vgl. Briesen (2020), 103-119. 
sibel ist, und dass sich alleine aus der Reaktionsabhängigkeit einer Eigenschaft keine Form des Bekanntschaftsprinzips ableiten lässt. Wenn überhaupt, so folgt das Prinzip also aus den Details einer reaktions-dispositionalen Analyse ästhetischer Eigenschaften. Anschließend werde ich in Abschnitt 3 einige Grundlagen klären: Was spricht eigentlich für eine reaktions-dispositionale Analyse ästhetischer Eigenschaften, und was genau heißt es, dass eine Eigenschaft in dieser Weise dispositional ist? In Abschnitt 4 werde ich dann auf Details der von mir favorisierten dispositionalen Theorie ästhetischer Eigenschaften eingehen. Abschließend werde ich in Abschnitt 5 auf dieser Basis eine schwache Version des Bekanntschaftsprinzips motivieren und auf Zusammenhänge im Hinblick auf den Urteilsbegriff in der Ästhetik zurückkommen.

\section{Bekanntschaftsprinzip und Reaktionsabhängigkeit}

Angenommen, du und ich seien in der Vergangenheit in Bezug auf 10000 unterschiedliche Gegenstände jeweils der gleichen Meinung hinsichtlich der Frage gewesen, ob der jeweilige Gegenstand schön sei. Nun berichtest du mir von einem mir unbekannten Gegenstand und beschreibst ihn als wunderschön. Wäre es in diesem Fall nicht plausibel, dass ich in der Annahme gerechtfertigt bin, der Gegenstand sei schön - ohne ihn selbst gesehen zu haben? Das Bekanntschaftsprinzip scheint also in der angegebenen Form zu stark formuliert zu sein. Ferner: In unseren Entscheidungen, welchen Film wir sehen, welche Ausstellung wir besuchen, welches Konzert wir hören wollen, orientieren wir uns häufig an den ästhetischen Einschätzungen anderer Personen. Doch wenn das Bekanntschaftsprinzip korrekt wäre, so wäre dieses Verhalten schwer zu erklären und eventuell sogar irrational.

Beide Probleme ließen sich durch eine Abschwächung des Prinzips umgehen:

Schwaches ästhetisches Bekanntschaftsprinzip

In den allermeisten Fällen ist die Überzeugung der Form „X ist schön/grazil/ elegant/etc.“ einer Person nur dann in hohem Maße gerechtfertigt, wenn die Person selbst den fraglichen Gegenstand $X$ gesehen hat.

In dieser Form ist das Prinzip nicht von vornherein unplausibel. Allerdings ist unklar, warum es gelten soll. Warum sollte in Bezug auf ästhetische Eigenschaften eine besondere epistemische Priorität der ersten Person gelten? Eine mögli- 
che Erklärung dafür könnte in der vermeintlich reaktions-dispositionalen Struktur ästhetischer Eigenschaften liegen.

Dispositionen sind Eigenschaften, die sich unter bestimmten Bedingungen manifestieren. Eine Flasche ist z. B. zerbrechlich, wenn sie über die Disposition verfügt, bei bestimmter Krafteinwirkung (Manifestationsbedingung) zu zerbrechen (Manifestation). Reaktions-dispositionale bzw. reaktionsabhängige Eigenschaften sind besondere Dispositionen, deren Manifestation in der mentalen Reaktion bewusster Subjekte besteht.

Folgt alleine aus der Reaktionsabhängigkeit einer Eigenschaft $F$, dass für „X $X$ ist $F$ “ eines der beiden Bekanntschaftsprinzipien gilt? Nein, denn es scheint durchaus möglich, (in vielen Fällen und in hohem Maße) gerechtfertigt zu glauben, dass einem Gegenstand eine bestimmte reaktions-dispositionale Eigenschaft zukommt, ohne dass sich diese Eigenschaft des Gegenstandes in mir selbst je manifestiert hat. Nehmen wir zu Illustrationszwecken dieses Punktes an, dass Farben reaktions-dispositionale Eigenschaften sind und z. B. Rot die Eigenschaft ist, in Personen unter bestimmten Wahrnehmungsbedingungen eine Roterfahrung auszulösen. ${ }^{3}$ Auch wenn ich diese Auffassung für korrekt halte, bin ich dadurch in keiner Weise auf die Annahme verpflichtet, dass ich nur dann (in hohem Maße) in der Überzeugung gerechtfertigt bin, die neuen Turnschuhe meines Sohnes seien rot, wenn ich sie selbst gesehen habe. Um gerechtfertigt zu glauben, dass seine Schuhe rot sind, ist es völlig ausreichend, wenn mein (generell vertrauenswürdiger) Sohn die Schuhe gesehen hat und mir von deren Farbe berichtet. In dieser Hinsicht scheint sich die vermeintlich reaktions-dispositionale Eigenschaft Rot nicht von anderen nicht-reaktions-dispositionalen Eigenschaften, z. B. der Schuhgröße, zu unterscheiden. Das Beispiel verdeutlicht, dass das ästhetische Bekanntschaftsprinzip, das eine besondere epistemische Priorität der ersten Person in Bezug auf ästhetische Eigenschaften behauptet, keineswegs alleine aus dem Umstand folgt, dass ästhetische Eigenschaften reaktionsdispositional sind.

Falls es also überhaupt einen interessanten Zusammenhang zwischen einer reaktions-dispositionalen Analyse ästhetischer Eigenschaften und dem Bekanntschaftsprinzip gibt, so hängt dies an den Details einer solchen Analyse. Bevor ich mich diesen Details zuwende, müssen noch einige Grundlagen geklärt werden.

3 Vgl. hierzu Johnston (1992). 


\section{Grundlegendes zu einer dispositionalen Analyse ästhetischer Eigenschaften}

Im Folgenden werde ich mich auf die ästhetische Eigenschaft (visueller) Schönheit konzentrieren. Was spricht für eine reaktions-dispositionale Auffassung von Schönheit? ${ }^{4}$ Betrachten wir die Aussage „X ist schön“, so fällt auf, dass sie weder rein subjektiv noch rein objektiv ist. Wer eine Schönheitsaussage für rein subjektiv hält, geht davon aus, dass wir mit einer solchen Aussage entweder nur unsere eigenen mentalen Zustände beschreiben oder einen solchen mentalen Zustand lediglich ausdrücken. Im ersten Fall wäre die Aussage „X ist schön“ synonym mit „Mir gefällt $X$ “ bzw. „Mir erscheint $X$ schön“; im zweiten Fall wäre sie eher vergleichbar mit Bravo-Rufen nach einer Theatervorstellung.

Gegen diese beiden subjektivistischen Positionen sprechen mehrere Punkte; zwei davon möchte ich ansprechen. Angenommen, ich äußere „X ist schön“, werde dann aber darauf hingewiesen, dass ich von mir unbemerkt bewusstseinsverändernde Drogen eingenommen habe, so scheint folgende sprachliche Reaktion angemessen: „Oh! Na gut, vielleicht ist $X$ nicht schön, aber $X$ erscheint mir gerade schön.“ Mit dieser Reaktion ziehe ich die ursprüngliche Aussage zurück und ersetze sie durch eine subjektive Aussage, in der ich explizit von meinem mentalen Zustand spreche. Wenn aber die ursprüngliche Aussage „X ist schön“ bereits synonym mit „Mir erscheint $X$ schön“ wäre, wäre die angeführte Reaktion weder angebracht noch hätte sie den genannten Effekt eines Rückzugs. Dieser Punkt lässt sich mit entsprechenden Anpassungen auch in Bezug auf die zweite oben angeführte subjektivistische Position anführen.

Ein weiterer Grund gegen rein subjektivistische Auffassungen kann anhand ästhetischer Meinungsverschiedenheiten verdeutlicht werden. Angenommen, du und ich seien uns in Bezug auf die Frage, ob $X$ schön sei, uneinig. Wenn du in diesem Fall auf meine Äußerung von „ $X$ ist schön“ mit „Nein, das ist falsch, $X$ ist nicht schön“ reagierst, so mag das unfreundlich und unpassend sein, aber sprachlich fehlerhaft ist es nicht. Allerdings wäre die Verwendung der expliziten Verneinung „Nein, das ist falsch“ sprachlich sehr wohl fehlerhaft, wenn der Satz „X ist schön“ synonym mit „Mir gefällt $X$ “ wäre. Warum? Angenommen, ich äußere „Mir gefällt $X$ “ so wäre deine Reaktion „Nein, das ist falsch, mir gefällt $X$ nicht“ deswegen fehlerhaft, weil $d u$ mit deiner Äußerung von ,..., mir gefällt $X$ nicht“ gar nicht die Proposition verneinst, die ich mit meiner Äußerung von „Mir gefällt $X$ “ ausdrücke, wodurch die explizite Verneinung „Nein, das ist falsch“ feh-

4 Vgl. zum Folgenden Briesen (2020), 61-95. 
lerhaft ist. Ein ähnlicher Punkt ließe sich auch gegen die zweite subjektivistische Option anführen, die „X ist schön“ analog zu Bravo-Rufen versteht.

Umgekehrt scheinen ästhetische Aussagen aber auch nicht vollständig objektiv zu sein. Wie - erstens - Frank Sibley überzeugend gezeigt hat, sind ästhetische Aussagen non-condition-governed: ${ }^{5}$ Aus einer wahren Aussage, dass ein Gegenstand über bestimmte objektive Eigenschaften verfügt, folgt nicht, dass er auch über bestimmte ästhetische Eigenschaften verfügt. Zweitens sprechen wir den unterschiedlichsten Gegenständen dieselbe ästhetische Eigenschaft zu. Eine Blume, ein Gebirgszug, eine Symphonie, ein Sofa, ein Gemälde etc. kann jeweils schön sein. Ist es wirklich plausibel anzunehmen, dass sich diese sehr unterschiedlichen Gegenstände eine relevante objektive (von uns unabhängige) Eigenschaft teilen? Ist es nicht viel plausibler anzunehmen, dass wir mit „X ist schön“ $X$ einer Klasse von Gegenständen zurechnen, die auf uns auf besondere Weise wirken? Es ist nicht verwunderlich, dass Dinge mit unterschiedlichen objektiven Eigenschaften in gleicher Weise auf uns wirken. Das Aroma echter Vanille und synthetisch hergestelltes Vanille-Aroma sind durch zwei sehr verschiedene chemische Substanzen realisiert, dennoch wirken beide Substanzen auf uns gleich oder zumindest sehr ähnlich. Weil es für uns von großem Interesse ist, wie bestimmte Substanzen und Gegenstände auf uns wirken, ist es auch nicht erstaunlich, dass wir eine Sprache mit Prädikaten entwickelt haben, die Gegenstände daraufhin klassifizieren, wie sie auf uns wirken - und es ist durchaus naheliegend, dass ästhetische Prädikate wie „schön“ in etwa diese Funktion übernehmen.

Es gibt also Gründe anzunehmen, dass ästhetische Aussagen der Form „X ist schön“ weder vollständig subjektiv noch vollständig objektiv sind. Die reaktionsdispositionale Analyse ästhetischer Eigenschaften kann das sehr gut erklären: Ästhetische Behauptungen sind nicht insofern subjektiv, als wir damit lediglich über unsere eigenen mentalen Zustände sprechen - vielmehr sprechen wir einem Gegenstand damit eine Eigenschaft zu. Diese Eigenschaft ist jedoch keine vollständig objektive, sondern eine reaktions-dispositionale Eigenschaft - d. h., es ist die Eigenschaft, auf uns auf bestimmte Weise zu wirken.

Man kann reaktions-dispositionale Eigenschaften vorläufig wie folgt spezifizieren:

Wenn $F$ eine reaktions-dispositionale Eigenschaft ist, dann ist folgendes (substantielle) Bikonditional (notwendigerweise) wahr:

5 Vgl. Sibley (1959). 
(BI) $X$ ist $F$ gdw. $X$ die Disposition zukommt, in Subjekten unter (tatsächlichen) Normalbedingungen $B$ die mentale Reaktion $M$ hervorzurufen.

Warum das Bikonditional nicht hinreichend für reaktions-dispositionale Eigenschaften ist und was mit den Ergänzungen in Klammern jeweils gemeint ist, kann hier vernachlässigt werden. ${ }^{6}$ Allerdings ist es hilfreich, einige Merkmale des Bikonditionals an einem Beispiel zu erläutern, ohne direkt in Schwierigkeiten ästhetischer Eigenschaften verwickelt $\mathrm{zu}$ werden. Daher gehe ich - wieder zu reinen Illustrationszwecken - davon aus, dass Farbeigenschaften reaktionsdispositional sind.

Wenn wir (BI) auf die Farbe Rot anwenden, so erhalten wir:

$X$ ist rot gdw. $X$ die Disposition zukommt, in Subjekten unter Normalbedingungen $B_{R}$ die mentale Reaktion $M_{R}$ hervorzurufen.

Um nun die Eigenschaft der Röte von anderen Farbeigenschaften abzugrenzen, muss einerseits $M_{R}$ näher bestimmt werden, wobei wir $M_{R}$ einfach als eine phänomenale Roterfahrung bestimmen können. Die Farbe Rot unterscheidet sich von Grün dadurch, dass es eine Roterfahrung statt einer Grünerfahrung auslöst. Dies verdeutlicht zweierlei: Um eine reaktions-dispositionale Eigenschaft zu individuieren, müssen wir erstens auf einen spezifischen mentalen Zustand bewusster Subjekte rekurrieren. Um diesen mentalen Zustand als Roterfahrung zu bestimmen, müssen wir zweitens auf den Terminus „rot“ Bezug nehmen. Obige Analyse sollte daher nicht so verstanden werden, als stellte die jeweils rechte Seite des Bikonditionals eine reduktive Analyse des Terminus auf der linken Seite dar.

Für die Individuierung der Eigenschaft Rot muss andererseits auch die Manifestationsbedingung $B_{R}$ spezifiziert werden. Eine farblose Pille, die in uns, wenn wir sie schlucken, eine Roterfahrung hervorruft, ist deswegen nicht rot. Nur wenn der fragliche Gegenstand auf unseren Gesichtssinn wirkt und dadurch eine Roterfahrung auslöst, ist der Gegenstand rot. Die reaktions-dispositionale Eigenschaft der Röte kann demnach scheinbar nur durch eine Kombination von Manifestationsbedingung und Manifestation individuiert werden. Dies gilt auch für ästhetische Eigenschaften.

Ein letzter Punkt, bevor ich mich der Analyse von Schönheit zuwende: Warum ist oben in Bezug auf $B_{R}$ von „Normalbedingungen“ die Rede? Der Verweis auf Normalbedingungen stellt eine Form der Idealisierung dar. Ein roter Gegenstand löst in Subjekten nur dann eine Roterfahrung aus, wenn die Wahrneh-

6 Vgl. aber Wedgewood (1998); Garcia-Carpintiero (2007); Briesen (2020), 139-178. 
mungsbedingungen in gewisser Hinsicht idealisiert sind: Die Wahrnehmung des Gegenstandes findet statt bei (a) annähernd Tageslicht, (b) aus angemessener Entfernung, (c) ohne relevante physiologische Einschränkung auf Seiten der Wahrnehmenden (z. B. Farbenblindheit) etc. Bei der Eigenschaft Rot kann diese Idealisierung - zumindest im Prinzip - einfach statistisch verstanden werden: Es handelt sich um die Bedingungen, die meistens vorliegen, wenn Menschen Farben wahrnehmen. Bei der reaktions-dispositionalen Analyse von Schönheit wird sich hingegen gleich ergeben, dass man die fragliche Idealisierung mit Sicherheit nicht statistisch einholen kann.

\section{Eine reaktions-dispositionale Bestimmung von Schönheit}

Wenn wir (BI) auf die Eigenschaft der Schönheit übertragen, so ergibt sich:

$X$ ist schön gdw. $X$ die Disposition zukommt, in Subjekten unter Normalbedingungen $B_{S}$ die Reaktion $M_{S}$ auszulösen.

Wie müssen in diesem Fall $B_{S}$ und $M_{S}$ bestimmt werden? Die Manifestation ästhetischer Eigenschaften $M_{S}$ hat eine deutlich komplexere Struktur als die Manifestation von Farbeigenschaften. Sie scheint sich aus mehreren Komponenten zusammenzusetzen - nämlich aus (I) einer bestimmten phänomenalen Erlebnisqualität, (II) einer bestimmten intentional gerichteten Emotion und (III) einer nicht-intentional gerichteten Stimmung. ${ }^{7}$

$\mathrm{Zu}$ (I): Der von schönen Gegenständen ausgelöste mentale Zustand $M_{S}$ lässt sich zunächst nur als phänomenale Schönheits-Erfahrung bestimmen. Ähnlich wie eine Roterfahrung nur unter Rekurs auf den Terminus „rot“ als solche bestimmt werden kann, lässt sich die besondere phänomenale Qualität der Erfahrung von etwas Schönem nur unter Rekurs auf den Terminus „schön“ bestimmen. Weil die Bestimmungen von reaktions-dispositionalen Eigenschaften nicht als reduktive Analysen der jeweiligen Termini „,rot“ bzw. „schön“ intendiert sind, wirft diese Zirkularität kein ernstes Problem auf.

$\mathrm{Zu}$ (II): Schönheit ist im Gegensatz zu Farbeigenschaften eine offensichtlich evaluative Eigenschaft. D. h., die Manifestation $M_{s}$ der fraglichen Disposition umfasst einen emotionalen Aspekt, den wir als Wohlgefallen spezifizieren

7 Vgl. zum Folgenden Briesen (2020), 181-218. 
können. Wohlgefallen ist eine Emotion, die auf ein bestimmtes Objekt gerichtet ist; es handelt sich um Wohlgefallen an etwas. Das Objekt, auf das das Wohlgefallen gerichtet ist, ist die in (I) angesprochene Erlebnisqualität. Wir genießen dieses Erlebnis, empfinden es in gewisser Hinsicht als lustvoll. Dieses Wohlgefallen geht dann auch mit einer Wertschätzung des Gegenstandes einher, der das phänomenale Erlebnis auslöst. $\mathrm{Zu} M_{S}$ gehören daher zwei objektbezogene Emotionen: Wohlgefallen an der besonderen phänomenalen Erfahrung und Wertschätzung des Gegenstandes, der die Erfahrung auslöst. Wobei wir das Wohlgefallen an der phänomenalen Erfahrung um ihrer selbst willen empfinden und den Gegenstand deswegen wertschätzen, weil er diese Erfahrung auslöst - nicht, weil er vorteilhaft in lebenspraktische Mittel-Zweck-Verhältnisse eingebettet wäre.

$\mathrm{Zu}$ (III): Neben den angesprochenen Aspekten umfasst die Erfahrung schöner Gegenstände einen weiteren gefühlsmäßigen Zustand, der sich aufgrund seiner nicht-intentionalen Charakteristik am besten als Stimmung verstehen lässt. Stimmungen wie Euphorie oder Traurigkeit sind von Emotionen wie Freude oder Trauer dadurch unterschieden, dass sie nicht auf spezifische Objekte gerichtet sind. Subjekte trauern um oder freuen sich auf bzw. über etwas. Das ist bei Stimmungen nicht der Fall. Das Stimmungsprofil, das ich in Bezug auf Schönheit im Blick habe, hängt eng mit den unter (I) und (II) angesprochenen Aspekten zusammen. Sowohl das Wohlgefallen an der Erlebnisqualität als auch die von MittelZweck-Verhältnissen unabhängige Wertschätzung des Gegenstandes tragen dazu bei, dass die Wahrnehmung schöner Gegenstände mit einer gewissen Stimmung des Enthobenseins von lebenspraktischen Verhältnissen einhergeht - auch wenn dieser Stimmungsaspekt so schwach und flüchtig sein kann, dass er kaum unsere bewusste Aufmerksamkeit erregt.

Damit ist die Manifestation der Disposition Schönheit für die Zwecke vorliegender Untersuchung hinreichend bestimmt. Wie lässt sich die Manifestationsbedingung $B_{S}$ spezifizieren? Zunächst ist festzuhalten, dass es sich hierbei, wie auch bei Farbeigenschaften, um Wahrnehmungsbedingungen handelt, die in gewisser Hinsicht idealisiert werden müssen. Allerdings werden die fraglichen Idealisierungen bei einer reaktions-dispositionalen Analyse von Schönheit wesentlich weitreichender ausfallen müssen. Warum?

Zunächst kann die Schönheit eines Gegenstandes auch von seiner Farbgebung abhängen, daher müssen wir alle bei Farben genannten Idealisierungen auch in Bezug auf Schönheit anführen: Die Wahrnehmung muss bei (a) annähernd Tageslicht, (b) aus angemessener Entfernung, (c) ohne relevante physiologische Einschränkungen auf Seiten des Subjekts (Farbenblindheit) etc. stattfinden. Allerdings hängt die phänomenale Erlebnisqualität (I) bei der Wahrnehmung eines schönen Gegenstandes nicht nur davon ab, dass ich den Gegenstand bei annähernd Tageslicht etc. betrachte, sondern auch davon, ob ich meine Aufmerk- 
samkeit in angemessener Weise auf die entsprechenden farblichen und formalen Merkmale des Gegenstandes richte. Diese Abhängigkeit lässt sich anhand sogenannter Wechselbilder verdeutlichen. Auf einem dieser Bilder sieht man je nach dem, worauf man die Aufmerksamkeit richtet, eine alte Frau mit großer Nase oder eine junge Frau mit Hut. Diese beiden Betrachtungsweisen gehen auch mit unterschiedlichen phänomenalen Erfahrungen einher. Etwas Ähnliches kann auch bei der Wahrnehmung von Gegenständen mit ästhetischen Eigenschaften eintreten. Zumindest bei Gegenständen, die komplexe visuelle Stimuli bieten, kann die besondere phänomenale Erlebnisqualität einer Schönheits-Erfahrung davon abhängen, dass Subjekte ihre Aufmerksamkeit auf entsprechende Aspekte des Gegenstandes richten.

Hinzu kommt, dass die Manifestation $M_{S}$ der reaktionsabhängigen Eigenschaft Schönheit einen Komplex aus (I) phänomenalen, (II) emotionalen sowie (III) stimmungsmäßigen Aspekten umfasst. Daher müssen die entsprechenden Wahrnehmungsbedingungen auch in der Hinsicht idealisiert werden, dass Umstände, die das Eintreten von (II) und (III) deutlich erschweren (oder vereinfachen) ausgeschlossen werden. Eine Person, die beispielsweise aufgrund bestimmter physiologischer oder psychologischer Störungen nicht in der Lage ist, Erfahrungen als lustvoll $\mathrm{zu}$ empfinden, wird bei der Wahrnehmung eines schönen Gegenstandes kein Wohlgefallen empfinden. Ebenso wird in einer Person, die so stark in lebenspraktische Mittel-Zweck-Überlegungen eingebunden ist, dass ihr Leben davon abhängt, bei der Erfahrung eines schönen Gegenstandes keine Stimmung des Enthobenseins aufkommen. All diese Umstände müssen daher im Rahmen einer Idealisierung aus den Wahrnehmungsbedingungen der reaktionsdispositionalen Eigenschaft der Schönheit ausgeschlossen werden.

Die Idealisierung der Manifestationsbedingungen ist damit aber noch nicht abgeschlossen. Konzentrieren wir uns für einen Moment auf Artefakte. Aus interessanten Überlegungen von Kendall Walton lässt sich entnehmen, dass die Frage, ob einem Artefakt eine bestimmte ästhetische Eigenschaft zukommt, von der angemessenen Vergleichsklasse abhängt. ${ }^{8}$ Dies lässt sich an einem Beispiel der Kunst gut erläutern. Dem Gemälde Broadway Boogie Woogie von Piet Mondrian kommen die ästhetischen Eigenschaften zu, dynamisch und energetisch zu sein. Dem werden allerdings nur Subjekte zustimmen, die als Vergleichsklasse die vom Künstler intendierte Klasse der De-Stijl-Bilder im Blick haben. Hat man jedoch als Vergleichsklasse nicht die Bilder der De-Stijl-Bewegung, sondern z. B. des abstrakten Expressionismus im Sinn, so erscheint einem Broadway Boogie Woogie nicht dynamisch, sondern eher statisch.

8 Vgl. Walton (1970). 
Dieses Beispiel genügt, um darauf hinzuweisen, dass die Wahrnehmungsbedingungen in Bezug auf ästhetische Eigenschaften in weiterer Hinsicht idealisiert werden müssen: Bei Artefakten müssen etwa auf Seiten des Subjekts hinreichend Kenntnisse in Bezug auf die Intentionen der Hersteller^innen sowie auf etwaige Entstehungskontexte vorliegen. Davon kann es abhängen, ob das Vorliegen der ästhetischen Eigenschaft in dem fraglichen Subjekt auch den dazugehörigen mentalen Zustand auslöst.

Zusammenfassend können wir festhalten, dass die Wahrnehmungsbedingungen bei ästhetischen Eigenschaften, wie z. B. der Eigenschaft der Schönheit, deutlich stärker idealisiert werden müssen als bei anderen mutmaßlich reaktionsabhängigen Eigenschaften wie z. B. Farben. Im Gegensatz zu Farbeigenschaften lassen sich diese Idealisierungen auch nicht statistisch einholen - einfach als die Bedingungen, die meistens vorliegen. Daher werde ich im Folgenden statt von „Normalbedingungen“ explizit von „Idealbedingungen“ sprechen:

$X$ ist schön gdw. $X$ die Disposition zukommt, in Subjekten unter Idealbedingungen $B_{S}$ die Reaktion $M_{S}$ auszulösen.

Zwei Bemerkungen sind für den weiteren Gang der Überlegung wichtig. Erstens: Die Liste an Idealisierungen enthält einige Punkte, die am ehesten aus erstpersonaler Perspektive beurteilt werden können. Auf welchen Aspekt richte ich meine Aufmerksamkeit? In welcher psychologischen Verfassung bin ich? Welche Kenntnisse habe ich über bestimmte Entstehungszusammenhänge des Gegenstandes? etc. Zweitens: Versuche, die oben angeführte Liste an Idealisierungen abschließend aufzuzählen, sind vermutlich aussichtslos. Man kann sich immer weitere Umstände ausdenken, in denen ein schöner Gegenstand bei einem wahrnehmenden Subjekt eben nicht den spezifizierten mentalen Zustand auslöst - und dieser Umstand müsste dann wieder im Rahmen einer Idealisierung ausgeschlossen werden. Die Aussichtslosigkeit, die Liste an Idealisierungen abzuschließen, ist jedoch kein schwerwiegendes Problem. Nur weil die Liste im Rahmen der Theorie nicht abschließend festgelegt werden kann, ist die oben angeführte Rede von Idealbedingungen keineswegs nichts-sagend und theoretisch fruchtlos. Denn für Personen, die den Terminus „schön“ verstehen, gilt: Wenn der Person eine Wahrnehmungssituation vollständig präsentiert würde (d. h., ihr alle Umstände der Situation - außer, ob ideale Bedingungen vorherrschen - bekannt wären), so wäre sie in der Lage zu entscheiden, ob in der fragliche Situation ideale Bedingungen vorliegen oder nicht. 


\section{Konsequenzen für das Bekanntschaftsprinzip und den Urteilsbegriff}

Was folgt aus der angeführten Analyse für die Rechtfertigung ästhetischer Überzeugungen? Zunächst gilt: Um gerechtfertigt zu glauben, dass $X$ schön ist, muss man - gemäß der angeführten Analyse - gerechtfertigt sein, dass $X$ über die Disposition verfügt, in Subjekten unter Idealbedingungen $B_{S}$ die Reaktion $M_{S}$ auszulösen. Doch wie lässt sich eine solche Annahme rechtfertigen? Die einfachste Möglichkeit wäre, sich selbst in eine Wahrnehmungssituation im Hinblick auf $X$ $\mathrm{zu}$ begeben und zu prüfen, ob sich der fragliche mentale Zustand $M_{S}$ bei einem selbst einstellt. Dies ist deswegen am einfachsten, weil einige Punkte im Hinblick auf die Idealisierungen der Situation am ehesten aus erstpersonaler Perspektive einschätzbar sind.

Wäre es auch möglich, in der Annahme, dass $X$ schön ist, dadurch gerechtfertigt zu sein, dass mir jemand die objektiven Eigenschaften des Gegenstandes beschreibt? Wenn die oben angeführte These Sibleys korrekt ist, dass ästhetische Urteile non-condition-governed sind (siehe Abschnitt 3) - es also aus keiner objektiven Beschreibung eines Gegenstandes folgt, dass er schön ist - so scheint die Chance auf eine solche Rechtfertigung eher gering.

Doch schließt das auch die Möglichkeit von testimonialer Rechtfertigung anderer Art aus? Angenommen, die Person beschreibe mir nicht die objektiven Eigenschaften des fraglichen Gegenstands, sondern sie äußerte mir gegenüber einfach: „X ist schön.“ Bei anderen mutmaßlich reaktions-dispositionalen Eigenschaften - nämlich Farbeigenschaften - gibt mir das durchaus einen Grund, die fragliche Aussage für wahr zu halten. Ich kann gerechtfertigt sein, dass die Schuhe meines Sohnes rot sind, weil er sie gesehen und es mir gesagt hat. Warum sollte dies bei ästhetischen Eigenschaften anders sein?

Dies lässt sich unter Rekurs auf die angeführte dispositionale Analyse von Schönheit durch dreierlei Hinweise beantworten. Erstens: Die jeweiligen Manifestationsbedingungen unterscheiden sich stark in ihrem Grad an Idealisierung. Auch bei Farben mussten diese Bedingungen idealisiert werden, aber dort konnte die Idealisierung statistisch eingeholt werden. Wenn uns daher jemand von der Farbe eines Gegenstandes berichtet, scheinen wir von vornherein in der Annahme gerechtfertigt zu sein, dass in der Wahrnehmungssituation der Person Normalbedingungen vorlagen. Daher können wir die entsprechende Überzeugung „X ist rot“ auch in epistemisch angemessener Weise aus zweiter Hand übernehmen. Anders verhält es sich, wenn uns eine Person von der Schönheit eines Gegenstandes berichtet. Die Manifestationsbedingungen von Schönheit $B_{s}$ haben in unserer Analyse viel stärkere und komplexere Idealisierungen erfahren, die 
nicht mehr statistisch als Normalbedingungen eingeholt werden können. Wir sind daher in diesem Fall nicht von vornherein gerechtfertigt anzunehmen, dass in den Wahrnehmungssituationen unserer Gesprächspartner`innen Idealbedingungen vorlagen.

Zweitens: In Bezug auf ästhetische Fragen sind Meinungsverschiedenheiten verbreiteter als in Bezug auf die Frage, welche Farbe einem Gegenstand zukommt. Wenn allerdings zwischen zwei Parteien Uneinigkeit darüber herrscht, ob ein bestimmter Gegenstand schön ist, so wird meist über die fragliche Wahrnehmungssituation diskutiert: Achten die Parteien auf unterschiedliche Aspekte des Gegenstandes; liegen unterschiedliche Kenntnisse in Bezug auf den Herstellungszusammenhang des Gegenstandes vor; gibt es weitere Umstände, in denen sich die Wahrnehmungssituationen jeweils unterscheiden? Dies legt nahe, dass in Bezug auf ästhetische Eigenschaften häufig eben keine Idealbedingungen vorliegen.

Drittens: Wir können im Rahmen der oben erläuterten testimonialen Gesprächssituation nicht durch Nachfragen eruieren, ob in der Wahrnehmungssituation unseres Gegenübers ideale Bedingungen vorlagen oder nicht. Denn wenn, wie oben gezeigt wurde, nicht alle Punkte auf der Liste an Idealisierungen abschließend aufgezählt werden können, dann lässt sich auch nicht jede möglicherweise relevante Idealisierung abfragen.

All dies führt nicht unbedingt dazu, dass wir in der ästhetischen Annahme „X ist schön“ niemals testimonial gerechtfertigt sind. Eine schwache Form der Rechtfertigung scheint unter gewissen Umständen durchaus aus zweiter Hand erworben werden zu können. Aber die angeführten Überlegungen verdeutlichen, dass Fälle, in denen wir durch das Zeugnis anderer ein hohes Maß an Rechtfertigung in Bezug auf „X ist schön“ erreichen können, äußerst seltene Ausnahmefälle bleiben dürften. Damit führt also die angeführte reaktions-dispositionale Analyse von Schönheit - insbesondere durch die genauere Spezifikation der Manifestationsbedingungen - zu einer Verteidigung des schwachen Bekanntschaftsprinzips.

\section{Schwaches ästhetisches Bekannschaftsprinzip (Schönheit)}

In den allermeisten Fällen ist die Überzeugung der Form „X ist schön“ einer Person nur dann in hohem Maße gerechtfertigt, wenn die Person selbst den fraglichen Gegenstand $X$ gesehen hat.

Welche Konsequenzen ergeben sich daraus für den Urteilsbegriff in der Ästhetik? In der Einleitung wurde in Bezug auf den Terminus „Urteil“ festgehalten, dass dessen Verwendung die bewusste und erstpersonale Auseinandersetzung mit den Gegenständen betont, über die ein Urteil gefällt wird. Außerdem wurde darauf hingewiesen, dass sich in der philosophischen Ästhetik der etwas altertümlich 
wirkende Begriff „Urteil“ unverändert halten konnte, obwohl er in anderen philosophischen Teildisziplinen größtenteils durch den Terminus „Überzeugung“ verdrängt wurde. Durch die angeführte Verteidigung des schwachen Bekanntschaftsprinzips konnte für den Bereich des Ästhetischen die epistemische Priorität erstpersonaler und selbstständiger Erfahrungen etabliert und begründet werden. Damit ergeben sich in Bezug auf den Urteilsbegriff in der Ästhetik zwei mögliche Erklärungs- bzw. Begründungsrichtungen. Erstens: Weil in der Ästhetik zumindest das schwache Bekanntschaftsprinzip gilt, so ist unter der Voraussetzung, dass der Urteilsbegriff tatsächlich mit einer Betonung des Erstpersonalen einhergeht, verständlich, warum in der Ästhetik größtenteils am Begriff des Urteils festgehalten wird. Zweitens gilt auch umgekehrt: Das Festhalten im Rahmen der Ästhetik am Urteilsbegriff spricht zusammen mit der Gültigkeit des schwachen Bekanntschaftsprinzips dafür, dass der Begriff des Urteils tatsächlich mit einer Betonung des Erstpersonalen, Eigenständigen und Selbstverantwortlichen verknüpft ist - ohne dass deswegen testimoniale ästhetische Rechtfertigung gänzlich ausgeschlossen werden muss.

\section{Literatur}

Briesen, J. (2020), Ästhetische Urteile und ästhetische Eigenschaften, Frankfurt am Main. Garcia-Carpintiero, M. (2007), A Non-modal Conception of Secondary Properties, in: Philosophical Papers 36.1, 1-33.

Johnston, M. (1992), How to Speak of the Colors, in: Philosophical Studies 68, 139-174. Kant, I. (2009), Kritik der Urteilskraft [1790], Hamburg. Robson, J. (2012), Aesthetic Testimony, in: Philosophy Compass 7.1, 1-10. Sibley, F. (1959), Aesthetic Concepts, in: The Philosophical Review 68, 421-450. Walton, K. (1970), Categories of Art, in: The Philosophical Review 79, 334-367. Wedgewood, R. (1998), The Essence of Response-Dependence, in: European Review of Philosophy 3, 31-54.

Wollheim, R. (1980), Art and its Objects, Cambridge. 